\title{
Personal User Interfaces for Recommender Systems
}

\author{
Martijn Millecamp \\ Department of Computer Science, KU Leuven \\ Leuven, Belgium \\ martijn.millecamp@cs.kuleuven.be
}

\author{
Katrien Verbert \\ Department of Computer Science, KU Leuven \\ Leuven, Belgium \\ katrien.verbert@cs.kuleuven.be
}

\begin{abstract}
In this Student Consortium submission, I outline the motivation and research questions of my $\mathrm{PhD}$ research on user interfaces for interacting with recommender systems, and particularly the interplay of different personal characteristics and effectiveness of different visualisation and interaction techniques. In addition, I describe the current status of my work and the planning of the next 2.5 years to complete my $\mathrm{PhD}$.
\end{abstract}

\section{CCS CONCEPTS}

- Human-centered computing $\rightarrow$ User studies; Information visualization; User models; User interface design; $\mathrm{Vi}$ sualization design and evaluation methods; • Social and professional topics $\rightarrow$ User characteristics; • Information systems $\rightarrow$ Personalization; Recommender systems.

\section{KEYWORDS}

recommender system; explanations; personal characteristics; music; Spotify; user characteristics; need for cognition

\section{ACM Reference Format:}

Martijn Millecamp and Katrien Verbert. 2019. Personal User Interfaces for Recommender Systems. In 24th International Conference on Intelligent User Interfaces (IUI '19 Companion), March 17-20, 2019, Marina del Ray, CA, USA. ACM, New York, NY, USA, 3 pages. https://doi.org/10.1145/1122445.1122456

\section{INTRODUCTION}

Recommender systems (RS) are increasingly used in our daily life, and offer great potential to help users find relevant items from within large collections. However, there are still some unresolved challenges that are withdrawing recommender systems to reach their full potential. One of these

Permission to make digital or hard copies of all or part of this work for personal or classroom use is granted without fee provided that copies are not made or distributed for profit or commercial advantage and that copies bear this notice and the full citation on the first page. Copyrights for components of this work owned by others than ACM must be honored. Abstracting with credit is permitted. To copy otherwise, or republish, to post on servers or to redistribute to lists, requires prior specific permission and/or a fee. Request permissions from permissions@acm.org.

IUI '19 Companion, March 17-20, 2019, Marina del Ray, CA, USA

(C) 2019 Association for Computing Machinery.

ACM ISBN 978-1-4503-9999-9/18/06 ..\$15.00

https://doi.org/10.1145/1122445.1122456 challenges is to increase the trust in the system by offering insight into the system and by enabling the user to steer the recommendation process [2]. Over the past decade, various studies attempted to resolve this challenge by providing explanations which enable users to explore the provenance of recommendations and/or by giving users control to steer the recommendation process. Despite the various attempts, almost none of these attempts has investigated the influence of personal characteristics (PC) e.g. experience, visual working memory, need for cognition, etc. on visual explanations and visual control techniques [1].

The goal of this research is to investigate the influence of PC on recommender system interfaces and to enable the development of such interfaces that can be adapted to individual user needs in a rich set of application domains. More specifically, we attempt to answer following research questions:

RQ1: Which specific PC may influence acceptance of recommendations?

RQ2: Are specific visualization techniques better than other techniques to enable user interaction with recommendation techniques? Does the answer to this question depend on specific PC or the application domain?

\section{RELATED WORK}

One of the approaches to make the RS more transparant and to enable the user to steer the recommendation process is by providing interactive visualizations [2]. Possible advantages of explaining recommendations are increasing user trust, efficiency, transparency, scrutability, effectiveness, persuasiveness and satisfaction [6]. For this research, we are most interested in transparent and scrutable explanations, as there is a lack of research about these kind of explanations [7].

In the field of recommender systems, there is a fair amount of research on PC, as well as on explanations. However, present methods mostly provide non-personalized explanations that are presented in an unstructured manner [5]. With our research, we would like to close this gap and research the influence of personal characteristics on visual, scrutable explanations and to provide guidelines for implementing personal explanations. 


\section{CURRENT STATUS}

To answer the research questions, we have been studying the effect of personal characteristics on the interaction with different recommender systems. So far, we conducted two user studies for which we implemented two different music recommender interfaces.

First user study. In a first user study, we compared the perception and the interaction of users with two different visual control techniques: sliders and a radar chart[4]. In an online, within-subject study, we asked the users to create two playlists: one with a (simple) interface providing sliders to steer the recommendation process and a second one where they could use the more advanced radar chart. Results showed that PC such as experience and visual working memory do not seem to play much role on the perception of the different control techniques, but that they do play a role on the interaction. In particular, higher experience (musical sophistication and Spotify usage) results in more interactions with the radar chart. In addition, a quarter of the participants reported that they liked the radar chart as it is better at displaying an overview of the attribute settings.

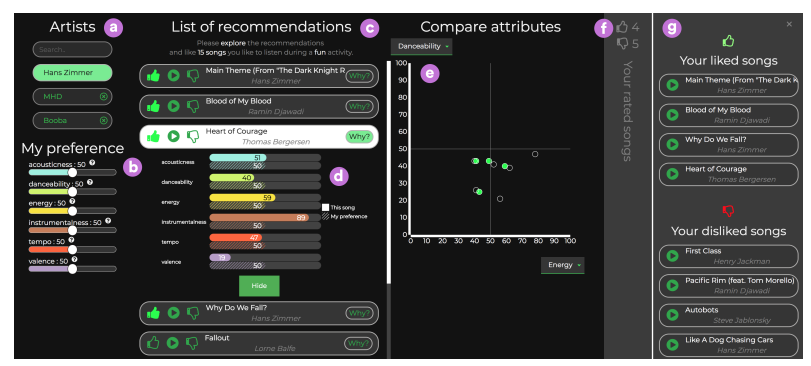

Figure 1: Interface of the second user study. [3]

Second user study. In a second user study, we researched the influence of PC on visual explanations of recommended songs. To do so, we conducted an online, within-subjects, study where all users where asked to make a playlist in both an interface with and without explained recommendations [3]. As Figure 1 shows, users could select an artist and modify six attributes as the seed for the recommendations (part a and b). In part c recommendations were explained by showing a grouped bar chart with both the preferred features of the user and the actual features of the song (part d). In addition, users could compare the different recommendations in a scatterplot (part e). Results showed that there was a significant interaction effect between the need for cognition score of a user and whether or not the interface provided explanations. This interaction effect was reported by users for expressing their confidence in the playlist they created. While users with a low score have more confidence in a playlist they created with an interface with explained recommendations, users with a high score have more confidence when the interface does not provide explanations.

\section{NEXT STEPS}

Based on these findings, we want to explore the influence of pc on explanations and control in a systematic way by conducting different online and offline user studies. So far, our user interfaces were designed on top of the Spotify API. Although interesting results have been obtained, we would like to research in a more systematic way what type of explanations and interaction features can be designed on top of different state-of-the recommendations algorithms.

As a first step, we are going to research the utility of different visualizations to explain and control recommendations generated by different state-of-the-art recommender systems. We also will research the influence of personal characteristics on these visualizations. To do so, we are implementing different user interfaces on top of different algorithms provided by the LensKit ${ }^{1}$ toolkit. This package provides state-of-the-art recommendations techniques, such as user- and item-based classical k-NN collaborative filtering and matrix factorization.

For each of these techniques, we want to implement different visual, scrutable explanations to help the users to steer the recommendation process. As our findings showed that the need and preference of different users is dependent on personal characteristics such as experience and need for cognition, we want to continue researching if the influence of these characteristics is independent of the recommendation technique.

To investigate these influences, we want to conduct both online and offline user studies. The goal of the online studies is to gather data to compare the perception and interaction of different users in the different interfaces. To gather this data, we can use existing crowd sourcing platforms. The overall objective is to collect a large sample size and infer data about potential relationship between personal characteristics and the effectiveness of different scrutable explanations.

The goal of the offline studies is to gain more insight in the users and to gather qualitative data. To gather this data, we are using eye-tracking to see the gaze pattern of users, semi-structured interviews to get more in-depth feedback of the users, and think-aloud data to gain insight in the user while he/she is using the interface.

The overall objective of both the online and offline user studies is to better understand which visualisation and interaction techniques work better for different recommendation algorithms and for different users. Both personal and situational characteristics, such as the application domain (recommending music, movies, news versus more high risk

\footnotetext{
${ }^{1}$ https://lenskit.org/
} 
recommendations related to health and well-being), will be collected to infer insight into the utility of different techniques for different users. At the end of this $\mathrm{PhD}$, we want to elaborate guidelines as well as open source visualisation libraries that can be used by both practitioners and researchers to develop scrutable interfaces on top of different recommendation algorithms. Insights from the user studies will help to select suitable techniques for the target audience of interactive recommender systems.

\section{ACKNOWLEDGMENTS}

Part of this research has been supported by the KU Leuven Research Council (grant agreement C24/16/017).

\section{REFERENCES}

[1] Fabian Greb, Wolff Schlotz, and Jochen Steffens. 2018. Personal and situational influences on the functions of music listening. Psychology of Music (2018).
[2] Chen He, Denis Parra, and Katrien Verbert. 2016. Interactive recommender systems: A survey of the state of the art and future research challenges and opportunities. Expert Systems with Applications 56 (2016), 9-27.

[3] Martijn Millecamp, Nyi Nyi Htun, Cristina Conati, and Katrien Verbert. 2019. To Explain or not to Explain: the Effects of Personal Characteristics when Explaining Music Recommendations. In Proceedings of the 2019 Conference on Intelligent User Interface. ACM, 1-12.

[4] Martijn Millecamp, Nyi Nyi Htun, Yucheng Jin, and Katrien Verbert. 2018. Controlling Spotify recommendations: effects of personal characteristics on music recommender user interfaces. In UMAP'18. ACM, 101-109.

[5] Sidra Naveed, Tim Donkers, and Jürgen Ziegler. 2018. ArgumentationBased Explanations in Recommender Systems: Conceptual Framework and Empirical Results. In UMAP'18. ACM, 293-298.

[6] Nava Tintarev and Judith Masthoff. 2007. A survey of explanations in recommender systems. In Data Engineering Workshop, 2007 IEEE 23rd International Conference on. IEEE, 801-810.

[7] Yongfeng Zhang and Xu Chen. 2018. Explainable Recommendation: A Survey and New Perspectives. arXiv preprint arXiv:1804.11192 (2018). 